(CC BY 4.0) | ISSN 2525-3409 | DOI: http://dx.doi.org/10.33448/rsd-v9i4.2878

Diferentes tipos de matéria orgânica no ensaio de reprodução da minhoca californiana

\title{
Eisenia andrei
}

Different types of organic matter in the reproduction test of the Californian earthworm

Eisenia andrei

Diferentes tipos de materia orgánica en la prueba de reproducción de la lombriz californiana Eisenia andrei

Recebido: 20/02/2020 | Revisado: 02/03/2020 | Aceito: 03/03/2020 | Publicado: 11/03/2020

Gabriel Eberl da Fonseca

ORCID: https://orcid.org/0000-0002-9106-3773

Universidade Federal do Rio Grande, Brasil

E-mail: gabriel.eberl@gmail.com

Rodrigo de Lima Brum

ORCID: https://orcid.org/0000-0002-0991-7347

Universidade Federal do Rio Grande, Brasil

E-mail: rodrigo.brum.93@gmail.com

Eduarda de Moura Garcia

ORCID: https://orcid.org/0000-0002-4075-8233

Universidade Federal do Rio Grande, Brasil

E-mail: eduarda11garcia@gmail.com

Laiz Coutelle Honscha

ORCID: https://orcid.org/0000-0003-4546-3642

Universidade Federal do Rio Grande, Brasil

E-mail: laizhonscha8@hotmail.com

Caroline Lopes Feijo Fernandes

ORCID: https://orcid.org/0000-0002-3931-9786

Universidade Federal do Rio Grande, Brasil

E-mail: carolinefernandesbio@gmail.com

Fernanda Oliveira Reis

ORCID: https://orcid.org/0000-0001-8086-6370

Universidade Federal do Rio Grande, Brasil

E-mail: fernanda.biotoxico@yahoo.com.br

Flavio Manoel Rodrigues da Silva Júnior 
ORCID: https://orcid.org/0000-0002-7344-4679

Universidade Federal do Rio Grande, Brasil

E-mail: f.m.r.silvajunior@gmail.com

\title{
Resumo
}

O presente estudo teve como principal objetivo avaliar a influência de diferentes tipos de matéria orgânica na toxicidade relativa ao rendimento reprodutivo de minhocas Eisenia andrei por meio do protocolo $\mathrm{n}^{\circ} 222$ da Organização para a Cooperação e Desenvolvimento Econômico (em inglês, OECD) - Teste de Reprodução de Minhocas. Foram analisadas 7 diferentes formulações de solo com diferentes fontes de matéria orgânica em diferentes porcentagens: Solo Artificial recomendado pela OECD com 10\% e $5 \%$ de matéria orgânica (esfagno); Solo Artificial Tropical (SAT) com 10\% e 5\% de matéria orgânica (fibra de coco), Solo Artificial com Café + Mate a $10 \%$ e $5 \%$ e húmus de minhoca em tratamentos com e sem ácido bórico (controle positivo e negativo, respectivamente). Os ensaios foram realizados com 5 réplicas contendo 10 minhocas cliteladas da espécie Eisenia andrei. A concentração de ácido bórico utilizada foi de $281 \mathrm{mg} \cdot \mathrm{kg}^{-1}$. O Solo Artificial OECD com 10\% de matéria orgânica teve melhor desempenho $(p<0,05)$. Desta forma, conclui-se que para as condições utilizadas no estudo este é o melhor substrato para realização do teste de rendimento reprodutivo em Eisenia andrei, com a turfa de esfagno como fonte de matéria orgânica, em relação aos demais testados.

Palavras-chave: Solo Artificial; Solo Artificial Tropical; Poluição do Solo; Oligoquetas.

\begin{abstract}
The present study aimed to evaluate the influence of different types of organic matter on reproductive toxicity of earthworms Eisenia andrei by means of Organization for Economic Cooperation and Development (OECD) Test No. 222 - Earthworm Reproduction Test. Seven different soil formulations with different sources of organic matter in different percentages were analyzed: Artificial Soil recommended by OECD with $10 \%$ and $5 \%$ organic matter (sphagnum); Tropical Artificial Soil with $10 \%$ and 5\% organic matter (fiber coconut); Artificial Soil with Coffee + Mate 10\% and 5\%; and earthworm humus in treatments with and without boric acid (positive and negative control, respectively). The assays were performed with 5 replicates containing 10 adult earthworm Eisenia andrei. The boric acid concentration used was 281 mg.kg-1. OECD Artificial Soil with $10 \%$ organic matter had better performance
\end{abstract}


( $p<0.05$ ). Thus, it is concluded that for the conditions used in the study this is the best substrate for performing the reproductive performance test in Eisenia andrei, with sphagnum peat as a source of organic matter, in relation to the others tested.

Keywords: Artificial Soil; Tropical Artificial Soil; Soil Pollution; Oligochaetes.

\section{Resumen}

El presente estudio tuvo como objetivo principal evaluar la influencia de diferentes tipos de materia orgánica en la toxicidad relacionada con el rendimiento reproductivo de las lombrices de tierra Eisenia andrei a través del protocolo 222 de la Organización para la Cooperación y el Desarrollo Económico (en inglés, OCDE) - Prueba de reproducción de lombrices de tierra. Se analizaron siete formulaciones de suelo diferentes con diferentes fuentes de materia orgánica en diferentes porcentajes: suelo artificial recomendado por la OCDE con 10\% y 5\% de materia orgánica (sphagnum); Suelo Artificial Tropical (SAT) con 10\% y 5\% de materia orgánica (fibra de coco), Suelo Artificial con Café + Mate al 10\% y 5\% y humus de lombriz en tratamientos con y sin ácido bórico (control positivo y negativo, respectivamente). Las pruebas se llevaron a cabo con 5 réplicas que contenían 10 lombrices de tierra de la especie Eisenia andrei. La concentración de ácido bórico utilizada fue de $281 \mathrm{mg} . \mathrm{kg}-1$. El suelo artificial de la OCDE con $10 \%$ de materia orgánica se desempeñó mejor ( $\mathrm{p}<0.05$ ). Por lo tanto, se concluye que para las condiciones utilizadas en el estudio, este es el mejor sustrato para realizar la prueba de rendimiento reproductivo en Eisenia andrei, con turba de sphagnum como fuente de materia orgánica, en relación con los otros probados.

Palabras clave: Suelo Artificial; Suelo Artificial Tropical; Contaminación del Suelo; Oligoquetos.

\section{Introdução}

O uso de bioensaios rápidos para detectar alterações causadas por agentes tóxicos no ambiente tem sido frequente em estudos de diagnóstico e monitoramento ambiental (Cotta et al., 2019). Para estes fins, os bioensaios precisam reunir algumas características, tais como: padronização, baixo custo, desfecho bem definido, além de ser suficientemente sensível para distinguir diferenças entre graus de contaminação distintos (Paton et al., 2005). Sendo assim, por meio dos ensaios ecotoxicológicos, são verificados os efeitos das variáveis ambientais capazes de afetar a toxicidade das substâncias disponíveis aos seres vivos de um ecossistema, podendo indicar uma resposta mais precisa da toxicidade dos contaminantes presentes nas 
amostras ambientais. Um dos métodos mais empregados para a avaliação dos efeitos adversos de agentes químicos sobre a biota terrestre e aquática são os testes de ecotoxicidade, onde são utilizados organismos-alvo terrestres, de águas continentais, estuarinas e marinhas que ficam expostos aos contaminantes sob condições controladas (CETESB, 2001).

Entre os organismos de solo, as minhocas ganham destaque, pois compreendem de $40 \%$ a $90 \%$ da biomassa de macrofauna da maioria dos ecossistemas tropicais (Fragoso et al., 1999) e têm sido o organismo mais utilizado para avaliação da qualidade do solo impactado por substâncias químicas. Estes organismos têm papel vital na decomposição de resíduos de plantas e ciclagem de nutrientes da matéria orgânica; na formação do húmus e de agregados de solo, onde a atividade biológica é mais intensa; no melhoramento da estrutura, fertilidade, porosidade e capacidade de infiltração, drenagem e retenção de água; e também no transporte de micro-organismos e nutrientes do solo por meio dos canais formados por sua escavação e deslocamento no solo (Ingham, 2006), podendo auxiliar também em processos de biorremediação de solos contaminados (Cotta, 2019).

Para invertebrados terrestres, um dos três grupos contemplados em normas técnicas padronizadas pela Associação Brasileira de Normas Técnicas (ABNT) é o grupo das minhocas: ABNT NBR 15537:2014 e ABNT NBR ISO 17512-1:2011. O primeiro protocolo refere-se ao teste de toxicidade aguda com minhocas, e o segundo para testes de fuga em Eisenia fetida e Eisenia andrei, ambos servindo para avaliar a qualidade do solo. No entanto, não existe uma norma padronizada nacional para a realização dos ensaios de reprodução, portanto foi utilizada a norma da Organização para a Cooperação e Desenvolvimento Econômico No. 222 (OECD, 2004). Vale destacar que esta norma utiliza Solo Artificial com uma composição diferente do solo utilizado no Brasil. No país, o solo utilizado é uma adaptação à realidade nacional (já incorporado nas normas mais recentes), chamado de Solo Artificial Tropical (SAT), onde a composição corresponde a $70 \%$ de areia fina, $20 \%$ de caulim e $10 \%$ de pó de fibra de coco, sendo este último em substituição ao "sphagnum" presente na norma da OECD.

Recentemente, outras adaptações têm sido propostas para o Solo Artificial, tal como a redução do conteúdo de pó de coco, considerando que os solos brasileiros têm baixa concentração de matéria orgânica (Assis, 2015). Além disso, é consenso que o solo artificial é pouco realístico e que não reflete as condições naturais do solo, porém mesmo com esta 
limitação o SAT é ainda amplamente utilizado em detrimento de bioensaios com solos naturais.

As características do solo podem ter grande influência na adsorção e consequentemente na biodisponibilidade e toxicidade de químicos no solo. Visto isso, os resultados do teste dependem das propriedades físico químicas intrínsecas do solo, que afetam o desempenho das espécies e a biodisponibilidade do agente químico em estudo (Kuperman et al., 2009). Comparando também os valores de CL50 entre um solo teste e um solo natural, a toxicidade da 3,4-dicloroanilina e do pentaclorofenol mostra-se maior quando em condição de solo natural. E isso provavelmente se dá por conta do seu menor conteúdo de matéria orgânica (Van Gestel, 1988), mas também outros fatores podem estar envolvidos.

A proteção bem-sucedida de comunidades exige conhecimento dos animais que compõem a base de cadeias tróficas e para isso faz-se necessário o conhecimento ecofisiológico sobre a susceptibilidade toxicológica de invertebrados de solo (Eijsackers et al., 1993). Desta forma, o objetivo do presente estudo é ampliar o conhecimento sobre as diferentes configurações de solo-teste propostas por diferentes agências normatizadoras (OECD e ABNT) por meio do ensaio de reprodução com Eisenia andrei comparando então os diferentes efeitos toxicológicos nas taxas de eficiência reprodutiva das minhocas testadas com a finalidade de poder indicar se há diferença expressiva entre o potencial de exibir a toxicidade dos diferentes solos em função de sua composição.

\section{Metodologia}

Para a realização deste trabalho, foram utilizadas espécimes da minhoca californiana Eisenia andrei adulta e com a presença de clitelo, mantidas em condições controladas de temperatura $\left(25^{\circ} \mathrm{C}\right)$, umidade $(40-50 \%)$ e fotoperíodo (16 claro: 8 escuro), em substrato de erva mate e borra de café, no Biotério de Invertebrados Terrestres do Instituto de Ciências Biológicas (ICB) da Universidade Federal de Rio Grande (FURG), localizada na cidade de Rio Grande, RS, Brasil..

Os tratamentos foram Solo Artificial 10\% - OECD (SA OECD 10\%), Solo Artificial 5\% - OECD (SA OECD 5\%), Solo Artificial Tropical Brasileiro 10\% (SAT BR 10\%), Solo Artificial Tropical Brasileiro 5\% (SAT BR 5\%), Solo Artificial Tropical - Mate + Café 10\% 
(SAT Mate+Café 10\%), Solo Artificial Tropical - Mate + Café 5\% (SAT Mate+Café 5\%) e húmus de minhoca (HM).

Solo Artificial 10\% - OECD (SA OECD 10\%): 10\% esfagno (o mais próximo do $\mathrm{pH}$ 5,5 ou 6,0, sem visíveis resquícios de plantas, moído e seco para a quantificação da umidade), $20 \%$ argila de caulim (contendo preferencialmente $30 \%$ de caulinita), entre 0,3 e 1,0 g de carbonato de cálcio ( $\mathrm{CaCO} 3$ pulverizado) para a obtenção inicial do $\mathrm{pH}$ de 6,0 \pm 0,5 e 70\% de areia de quartzo (dependendo da quantidade de $\mathrm{CaCO} 3$ requerida).

Solo Artificial 5\% - OECD (SA OECD 5\%): 5\% esfagno (o mais próximo do pH 5,5 ou 6,0, sem visíveis resquícios de plantas, moído e seco para a quantificação da umidade), $22,5 \%$ argila de caulim (contendo preferencialmente $30 \%$ de caulinita), entre 0,3 e 1,0 carbonato de cálcio (CaCO3 pulverizado) para a obtenção inicial do pH de 6,0 \pm 0,5, e 72,5\% de areia de quartzo (dependendo da quantidade de $\mathrm{CaCO} 3$ requerida).

Solo Artificial Tropical Brasileiro 10\% (SAT BR 10\%): 10\% fibra de coco (o mais próximo do $\mathrm{pH} 5,5$ ou 6,0, sem visíveis resquícios de plantas, moído e seco para a quantificação da umidade), entre 0,3 e 1,0 carbonato de cálcio ( $\mathrm{CaCO} 3$ pulverizado) para a obtenção inicial do pH de 6,0 $\pm 0,5$, e 70\% de areia de quartzo (dependendo da quantidade de $\mathrm{CaCO} 3$ requerida).

Solo Artificial Tropical Brasileiro 5\% (SAT BR 5\%): 5\% fibra de coco (o mais próximo do $\mathrm{pH} 5,5$ ou 6,0, sem visíveis resquícios de plantas, moído e seco para a quantificação da umidade), 22,5\% argila de caulim (contendo preferencialmente $30 \%$ de caulinita); entre 0,3 e 1,0 carbonato de cálcio ( $\mathrm{CaCO} 3$ pulverizado) para a obtenção inicial do $\mathrm{pH}$ de $6,0 \pm 0,5$, e 72,5\% de areia de quartzo (dependendo da quantidade de $\mathrm{CaCO} 3$ requerida).

Solo Artificial Tropical - Mate + Café 10\% (MATE+CAFE 10\%): 10\% mistura de rejeitos de erva mate e borra de café (o mais próximo do $\mathrm{pH}$ 5,5 ou 6,0, sem visíveis resquícios de plantas, moído e seco para a quantificação da umidade), 20\% argila de caulim (contendo preferencialmente $30 \%$ de caulinita); 0,3 e 1,0 carbonato de cálcio (CaCO3 pulverizado) para a obtenção inicial do $\mathrm{pH}$ de $6,0 \pm 0,5$, e $70 \%$ de areia de quartzo (dependendo da quantidade de $\mathrm{CaCO} 3$ requerida).

Solo Artificial Tropical - Mate + Café 5\% (MATE+CAFE 5\%): 5\% Mistura de rejeitos de erva mate e borra de café (o mais próximo do $\mathrm{pH} 5,5$ ou 6,0, sem visíveis 
resquícios de plantas, moído e seco para a quantificação da umidade), 22,5\% argila de caulim (contendo preferencialmente $30 \%$ de caulinita), 0,3 to 1,0 carbonato de cálcio $(\mathrm{CaCO} 3$ pulverizado) para a obtenção inicial do $\mathrm{pH}$ de $6,0 \pm 0,5$, e 72,5\% de areia de quartzo (dependendo da quantidade de $\mathrm{CaCO} 3$ requerida).

Húmus de minhoca (HUMUS): Foi utilizado em quintuplicata também o substrato total de húmus de minhoca comercial afim de comparar os mesmos parâmetros nesse solo considerado rico em nutrientes.

Para a execução do Protocolo OECD No. 222 (Teste de Reprodução de Minhocas) foi realizada a exposição de minhocas adultas e cliteladas que foram expostas durante 4 semanas a uma substância de referência (H3BO3 - Ácido Bórico) junto a um controle negativo de água destilada. Foi utilizada apenas a concentração de $281 \mathrm{mg} . \mathrm{kg}-1$ de ácido bórico baseando-se na CE50 determinada no trabalho de Niemeyer et al. (2018), que utilizou solo equivalente ao SAT BR 10\% (Solo artificial convencionalmente utilizado no Brasil). Todos os tratamentos foram realizados em quintuplicata, contendo 10 organismos por recipiente.

Assim, examinou-se os efeitos de mortalidade das minhocas adultas após 4 semanas de exposição que então foram removidas do solo com a finalidade de avaliar os efeitos na reprodução depois de mais 4 semanas de incubação nas mesmas condições da etapa anterior. $\mathrm{Na}$ etapa final, conta-se o número de proles presentes no solo. O rendimento reprodutivo das minhocas expostas ao teste químico foi comparado com o controle negativo e entre si para que então pudesse se classificar as diferentes matrizes de solo com relação à interferência na toxicidade de agentes químicos em função da sua composição de matéria orgânica.

O teste teve início a partir do momento em que as minhocas são adicionadas aos recipientes dos grupos contendo o solo contaminado com ácido bórico e aos grupos controle. Para todos os 14 tratamentos, bem como todas suas réplicas, a alimentação era realizada semanalmente através de restos de erva-mate e tendo sua água também reposta conforme o necessário. Ao final dos primeiros 28 dias, as minhocas adultas vivas são observadas e contadas. Após isso, todas foram removidas dos recipientes e pesadas.

Após a remoção das minhocas adultas, o solo com os casulos foi então incubado por quatro semanas adicionais sob as mesmas condições de teste como, temperatura, fotoperíodo e umidade, exceto as condições de alimentação que ocorreu apenas uma vez no início desta fase do teste. 
No final do segundo período de 4 semanas, o número de juvenis surgidos dos casulos no solo teste foi contado. Todos os sinais de danos às minhocas foram registrados ao longo do período do teste. Ao término do teste, os juvenis foram encaminhados para o processo de eutanásia.

Os valores biométricos gerados foram avaliados pelo Teste de Kolmogorov Smirnov para avaliar a normalidade dos dados e apresentados como média \pm erro padrão de número de juvenis em cada tratamento. Para comparação dos diferentes grupos experimentais foi realizada a análise de variância (ANOVA) seguida de um teste de Tukey $(\mathrm{p}<0,05)$. As comparações não pareadas entre o tratamento e seu respectivo controle positivo (ácido bórico) foram feitas a partir de teste $t$, considerando o $\mathrm{p}$ crítico como $\mathrm{p}<0,05$. Todos os testes foram realizados no software PRISM 5.0.

\section{Resultados e Discussão}

Os resultados do rendimento reprodutivo dos diferentes grupos experimentais estão mostrados nas Figura 1 (sem ácido bórico) e 2 (com ácido bórico). As diferenças estatísticas entre os grupos sem ácido bórico (controle negativo com água destilada) foram entre o solo padrão utilizado na norma OECD (SA OECD 10\%) e os demais tratamentos. Já nas comparações entre os controles positivos, expostos ao ácido bórico (H3BO3), houve apenas diferença entre os grupos SAT 10\% C+ e o grupo MATE+CAFÉ 5\% C+. 


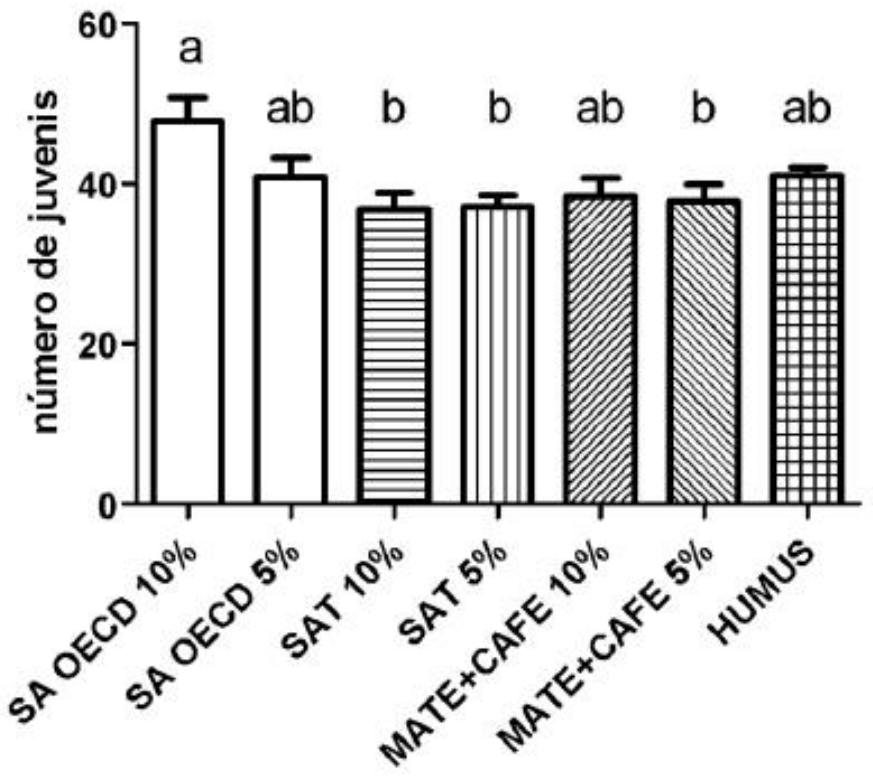

Figura 1. Comparação entre o número de juvenis ao fim de 56 dias dos grupos experimentais sem exposição ao ácido bórico.

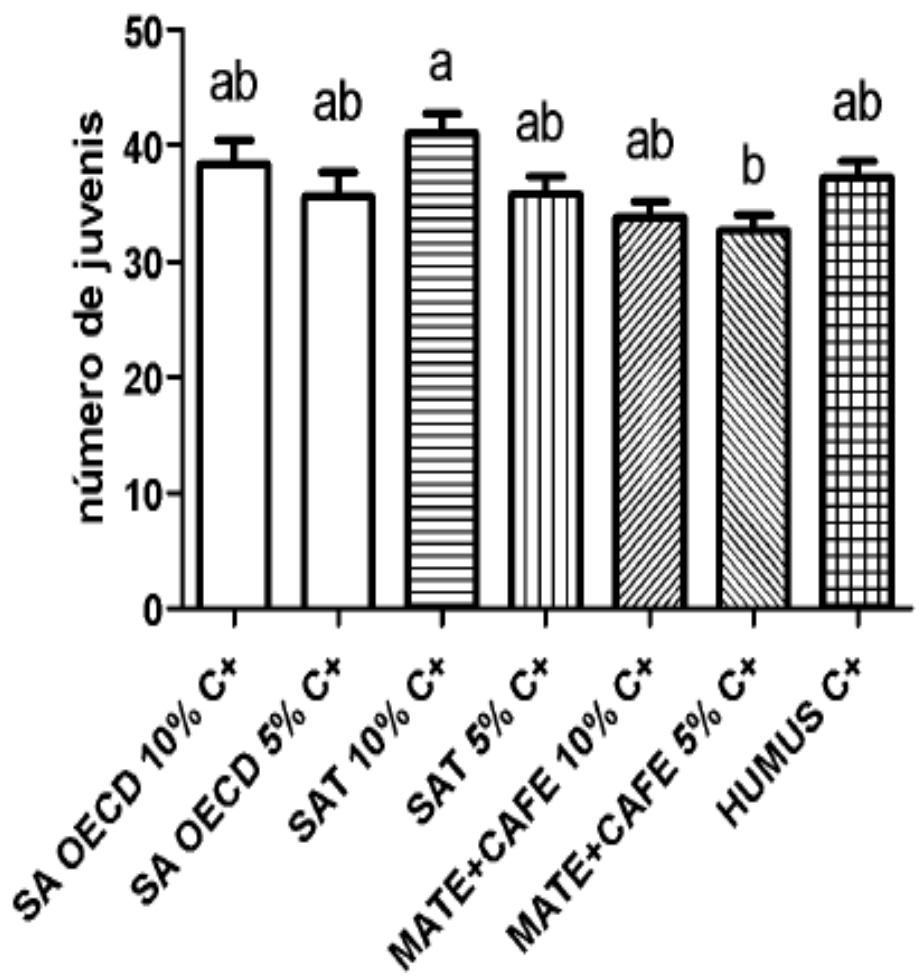

Figura 2. Comparação entre o número de juvenis ao fim de 56 dias dos grupos experimentais expostos ao ácido bórico. 
Para a Figura 1, bem como para a Figura 2, as letras atribuídas às colunas correspondem à suas diferenças estatísticas advindas da realização do teste Tukey.

A comparação entre os grupos sem exposição ao ácido bórico (controle negativo) e expostos ao ácido bórico (controle positivo) está mostrada na Tabela 1. Entre todos os grupos, houve diferença significativa entre os solos da norma padrão OECD No. 222 (grupo SA OECD 10\% C- versus SA OECD 10\% C+).

Tabela 1. Comparação entre a média de juvenis entre os grupos tratados com e sem ácido bórico.

\begin{tabular}{lccc}
\hline \multicolumn{1}{c}{ Tratamento } & $\begin{array}{c}\text { C- (sem ácido } \\
\text { bórico })\end{array}$ & $\begin{array}{c}\text { C+ (com ácido } \\
\text { bórico })\end{array}$ & Valor de $p$ \\
\hline SA OECD 10\% & $47,80 \pm 2,9$ & $38,40 \pm 2,1$ & 0,03 \\
SA OECD 5\% & $40,80 \pm 2,3$ & $35,60 \pm 2,1$ & 0,13 \\
SAT BR 10\% & $36,80 \pm 2,1$ & $41,00 \pm 1,7$ & 0,16 \\
SAT BR 5\% & $37,00 \pm 1,5$ & $35,80 \pm 1,5$ & 0,58 \\
MATE+CAFÉ 10\% & $38,40 \pm 2,3$ & $33,80 \pm 1,3$ & 0,12 \\
MATE+CAFÉ 5\% & $37,80 \pm 2,1$ & $32,60 \pm 1,4$ & 0,07 \\
HUMUS & $41,00 \pm 1,0$ & $37,20 \pm 1,4$ & 0,06 \\
\hline
\end{tabular}

Apesar das limitações do estudo quanto a avaliação apenas através de um único parâmetro/teste e com única espécie de organismo teste. Tais dados nos permitem supor que mesmo diante da influência das características físico-químicas do solo na toxicidade de agentes químicos apenas o solo artificial OECD com 10\% de matéria orgânica mostrou-se sensível quanto a biodisponibilizar o agente tóxico (ácido bórico) às minhocas Eisenia andrei.

No entanto, comparando esses resultados de toxicidade crônica no ensaio de reprodução com Eisenia fetida/Eisenia andrei, com outro trabalho que também utilizou o ácido bórico como agente químico testado, nota-se diferenças quanto à toxicidade do mesmo. No trabalho de Niemeyer et al. (2018), em que utilizou o SAT comum (equivalente ao SAT BR 10\% deste trabalho), há uma diferença na eficiência reprodutiva das minhocas demonstrada no número de juvenis ao fim do teste em relação ao presente trabalho. Segundo a curva de dose-resposta de trabalho em questão, na mesma concentração há uma média de 20 juvenis ao fim do teste. Uma variação considerável em relação ao presente estudo (acima de 
35 juvenis nas duas porcentagens de matéria orgânica). No entanto, é importante considerar que Niemeyer realizou comparações de resultados interlaboratoriais e indica que há variação considerável entre estes, justificando as variações em função de diferenças metodológicas, sensibilidade dos organismos e variações de ambiente de realização dos ensaios.

Ainda é de consenso na ecotoxicologia que as características físico químicas do meio influenciam na biodisponibilidade de agentes químicos. No entanto, Kuperman et al (2009) pontua que é questionável a relevância ecológica de algumas metodologias e abordagens aplicadas ainda hoje, visto que o estudo da ecotoxicologia terrestre tem pouco mais de duas décadas e desde então não houve muitas indagações sobre diferentes aspectos como a composição do solo, se essas matrizes de solo são recomendadas para todo tipo de agente químico, se as matrizes artificiais são fidedignas às condições de solo natural, entre outras dúvidas.

Os autores consideram também que para os avanços científicos futuros e a melhoria da relevância ecológica de dados empíricos para avaliações de solos contaminados exige-se: a transição da pesquisa ecotoxicológica dos testes artificiais baseados no solo para o uso de solos naturais para exposições de espécies; expandir a lista existente de espécies de teste, que foram selecionadas principalmente com base em considerações práticas, para incluir espécies que são geograficamente e ecologicamente representativas do local e das condições do local; e complementando testes laboratoriais de espécies únicas com avaliações da comunidade e do nível do ecossistema (Kuperman et al 2009).

Este posicionamento vai ao encontro do que Niva et al. (2016) abordam em uma recente revisão sobre aspectos ecotoxicológicos em solos brasileiros. Os autores reforçam a necessidade de considerar cenários mais realísticos de exposição, considerando as diferenças regionais, incluindo o uso de testes crônicos como o ensaio de reprodução. Quanto a padronização de ensaios de reprodução, Niemeyer et al. (2009) iniciaram esforços para investigar as respostas de reprodução em condições de laboratório do isópodo terrestre Cubaris murina, porém este seja talvez o único estudo nacional com este propósito.

\section{Conclusão}

Os resultados obtidos no presente estudo mostram que apenas o solo artificial OECED com $10 \%$ de matéria orgânica foi sensível quanto a disponibilizar o ácido bórico às minhocas. Conclui-se então que a recomendação atual, baseada nos resultados deste estudo e nas 
condições dos bioensaios aqui apresentadas, que o melhor substrato para realização do teste de rendimento reprodutivo em Eisenia andrei é o Solo Artificial proposto pela OECD, que possui em sua composição a turfa de esfagno como fonte de matéria orgânica, diferentemente das outras variações testadas. Mostrando assim que as diferentes configurações de solo-teste propostas por agências normatizadoras podem interagir de formas distintas sobre a reprodução da minhoca Eisenia andrei.

Sugerimos que as agências normatizadoras, sociedades científicas e órgãos afins busquem a realização de programas de comparação Inter laboratorial com consequente padronização desta norma, com vistas à uniformização de seu uso em território nacional. Por fim, diante da menor representatividade ecológica dos solos artificiais sugerimos como continuidade deste estudo a comparação dos solos artificiais com solos naturais de diferentes origens.

\section{Referências}

Associação Brasileira de Normas Técnicas. (2011). ABNT; NBR ISO 17512-1: Qualidade do Solo: Ensaio de Fuga para Avaliar A Qualidade de Solos e Efeitos de Substâncias Químicas no Comportamento. Parte 1: Ensaio com Minhocas (Eisenia Foetida e Eisenia Andrei).

Associação Brasileira de Normas Técnicas. (2014). Abnt; Nbr 15537: Ecotoxicologia Terrestre: Toxicidade Aguda - Método de Ensaio com Minhocas (Lumbricidae).

Assis, O. (2015). Enquitreídeos (Enchytraeidae, Oligochaeta) Como Indicadores do Manejo do Solo e em Ensaios Ecotoxicológicos. Dissertação de Mestrado. Universidade Tecnológica Federal do Paraná. Acesso em $19 \quad$ Fevereiro, em Http://Repositorio.Utfpr.Edu.Br/Jspui/Bitstream/1/1848/1/Ct_Ppgcta_M_Assis\%2c\%20orlan do_2015.Pdf

Companhia de Tecnologia de Saneamento Ambiental. Cetesb (2001). Relatório de Estabelecimento de Valores Orientadores para Solos e Águas Subterrâneas no Estado de São Paulo. São Paulo, Brasil. 
Cotta, J. A. O. (2019). Ácidos Húmicos: A Dinâmica de Mineralização Durante Biorremediação por Vermicompostagem de Solos Contaminados por Diesel. Eisenia Fétida. Research, Society and Development. 8 (8).

Cotta, J. A. O.; Lemos, G. S.; Lima, E. N. (2019). Ensaios Ecotoxicológicos de Solo Contaminado por Diesel Submetido à Degradação pela Espécie Eisenia Fétida. Research, Societya and Development. 9 (2).

Eijsackers, H.; Heimbach, F.; Donker, M. H. (1993). Ecotoxicology of Soil Organisms. Crc Press.

Fragoso, C.; Lavelle, P.; Blanchart, E.; Senapati, B. K.; Jimenez, J. J.; Martinez, M. A.; Decaëns, T.; Tondoh, J. (1999). Earthworm Communities of Tropical Agroecosystems: Origin, Structure And Influence Of Management Practices. Earthworm Management in Tropical Agroecosystems, 27-55.

Ingham, E. R. the Soil Biology Primer. Acesso em 26 Janeiro, em https://www.nrcs.usda.gov/wps/portal/nrcs/main/soils/health/biology/

Kuperman, R. G.; Checkai, R. T.; Garcia, M. V. B.; Römbke, J.; Stephenson, G. L.; Sousa, J. P. (2009). State of the Science and the Way Forward for the Ecotoxicological Assessment of Contaminated Land. Pesquisa Agropecuária Brasileira, 44(8), 811-824.

Niemeyer, J. C.; Santos, V. C.; Araújo, P. B. D.; Da Silva, E. M. (2009). Reproduction of Cubaris Murina (Crustacea: Isopoda) Under Laboratory Conditions and its use In Ecotoxicity Tests. Brazilian Journal of Biology, 69 (1), 137-142.

Niemeyer, J. C.; Carniel, L. S. C.; De Santo, F. B.; Silva, M.; Klauberg-Filho, O. (2018). Boric Acid as Reference Substance for Ecotoxicity Tests in Tropical Artificial Soil. Ecotoxicology, 27 (4), 395-401.

Niva, C. C.; Niemeyer, J. C.; Júnior, F. M. R. D. S.; Nunes, M. E. T.; De Sousa, D. L.; Aragão, C. W. S.; Sauter K. D.; Espindola E. G.; Sousa, J. P.; Römbke, J. (2016). Soil 
Ecotoxicology in Brazil is Taking its Course. Environmental Science and Pollution Research, 23(11), 11363-11378.

Organization For Economic Cooperation And Development. (2004). test no. 222: Earthworm Reproduction Test (Eisenia Fetida/Eisenia Andrei).

Paton, G. I.; Killham, K.; Weitz, H. J.; Semple, K. T. (2005). Biological Tools for the Assessment of Contaminated Land: Applied Soil Ecotoxicology. Soil use Management, 21, 487-499.

Van Gestel, C. A. M.; Van Dis, W. A. (1988). The influence of soil characteristics on the toxicity of four chemicals to the earthworm Eisenia fetida andrei (Oligochaeta). Biology and Fertility of Soils, 6(3), 262-265.

\author{
Porcentagem de contribuição de cada autor no manuscrito \\ Gabriel Eberl Da Fonseca - 30\% \\ Rodrigo De Lima Brum - 10\% \\ Eduarda De Moura Garcia - 10\% \\ Laiz Coutelle Honscha - 10\% \\ Caroline Lopes Feijo Fernandes - 10\% \\ Fernanda Oliveira Reis $-10 \%$ \\ Flavio Manoel Rodrigues Da Silva Júnior - 20\%
}

